\title{
AIAA-2002-1465
}

\section{Optimized Vertex Method and Hybrid Reliability}

Steven A. Smith, T. Krishnamurthy and B.H. Mason

NASA Langley Research Center

Hampton, VA

\section{$4^{\text {rd }}$ AIAA/ASME/ASCE/AHS/ASC Structures, Structural Dynamics, and Materials Conference \\ 22-25 April, 2002 \\ Denver, Colorado}

For permission to copy or republish, contact the American Institute of Aeronautics and Astronautics 1801 Alexander Bell Drive, Suite 500, Reston, VA 22091 


\title{
Optimized Vertex Method and Hybrid Reliability
}

\author{
Steven A. Smith *, T. Krishnamurthy ${ }^{\dagger}$ and B.H. Mason * \\ NASA Langley Research Center \\ Hampton, VA 23681-2199
}

\section{Abstract}

A method of calculating the fuzzy response of a system is presented. This method, called the Optimized Vertex Method (OVM), is based upon the vertex method but requires considerably fewer function evaluations. The method is demonstrated by calculating the response membership function of strainenergy release rate for a bonded joint with a crack. The possibility of failure of the bonded joint was determined over a range of loads. After completing the possibilistic analysis, the possibilistic (fuzzy) membership functions were transformed to probability density functions and the probability of failure of the bonded joint was calculated. This approach is called a possibility-based hybrid reliability assessment. The possibility and probability of failure are presented and compared to a Monte Carlo Simulation (MCS) of the bonded joint.

\section{Introduction!}

In the future, the consideration of variability in design parameters and the calculation of reliability will be required for aerospace structures. In the final stages of design it is clear that the evaluation of system reliability will require full probabilistic analysis. However, probabilistic analyses are computationally expensive and there may not be enough information to use probabilistic methods to assess reliability early in the design cycle. Hence, methods that require less information and yet can provide a measure of reliability are attractive for the early stages of design.

Possibility theory, a variant of fuzzy set

\footnotetext{
- Aerospace Engineer, Army Research Laboratory, Member AlAA

${ }^{\dagger}$ Aerospace Engineer, Analytical and Computational Methods Branch, Senior Member AIAA

* Aerospace Engineer, Analytical and Computational Methods Branch, Member AIAA
}

Copyright $\mathbb{C} 2002$ by the American Institute of Aeronautics and Astronautics, Inc. No copyright is asserted in the United States under Title 17, U.S. Code. The U.S. Government has a royalty-free license to exercise all rights under the copyright claimed herein for government purposes. All other rights are reserved by the copyright owner. theory, is one method that does not require detailed statistical data and can be used to assess reliability (Ref. 1,2). The vertex method (Ref. 3) has commonly been used to calculate the fuzzy response of a system. A disadvantage of the vertex method is that the number of function evaluations needed increases by the number of alpha-cuts raised to the power of the number random variables, where the number of alpha-cuts refers to the levels of possibility where calculations are performed. Without computational efficiency possibility theory becomes less attractive than probabilistic methods for assessing reliability.

Possibility theory has also been shown to be more conservative than probability for parallel systems or for single failure modes (Ref. 4,5). While a conservative solution is desired for the evaluation of structures, overly conservative designs generally carry a weight penalty. A solution that reduces the conservatism of possibility is also desirable.

This paper presents a modified vertex method that uses gradient-based optimization and is computationally efficient. The method is demonstrated for a simple function and then applied to a more complex structural problem. The structural example that is used to demonstrate the method is the bonded joint investigated earlier by Stroud et al. (Ref. 6). In Reference 6 the authors noted that the possibilistic analysis was conservative compared to the probabilistic analysis. To address the problem of the conservative nature of possibility theory, possibilistic membership functions are transformed to probability density functions for the calculation of the probability of failure.

\section{Calculating Possibility}

In Figure 1 an example of a possibilistic membership function is presented. A possibilistic membership function is sometimes called a fuzzy number or a fuzzy variable. One method for evaluating fuzzy relationships is the vertex method proposed by Dong and Shah (Ref. 3). In the vertex method, rather than discretizing the variable domain, the membership domain is discretized. The disretization of the membership domain is accomplished by dividing the membership domain into a series of equally spaced cuts, called $\alpha$-cuts, where $\alpha$ represents possibility. For 
each $\alpha$-cut the upper and lower value of the fuzzy variable is selected.

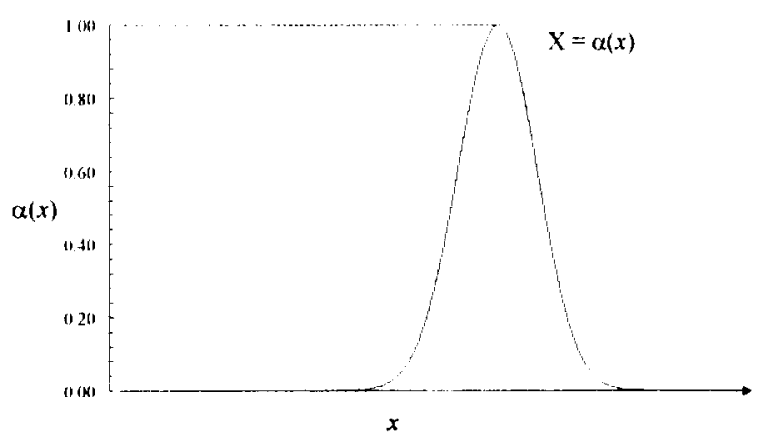

Figure 1. Membership function for a fuzzy number

For the membership function shown in Figure 2 at $\alpha=0$ the upper and lower bounds are the values $e_{\text {, }}$ and $a$. Suppose that $Z=f(X, Y)$, where $f(X, Y)$ is a fuzzy operation and $\mathrm{X}$ and $\mathrm{Y}$ are fuzzy numbers described by a membership functions similar to the one shown in Figure 2. The fuzzy numbers $X$ and $Y$ are described by their membership functions, $\alpha(x)$ and $\alpha(y)$, respectively.

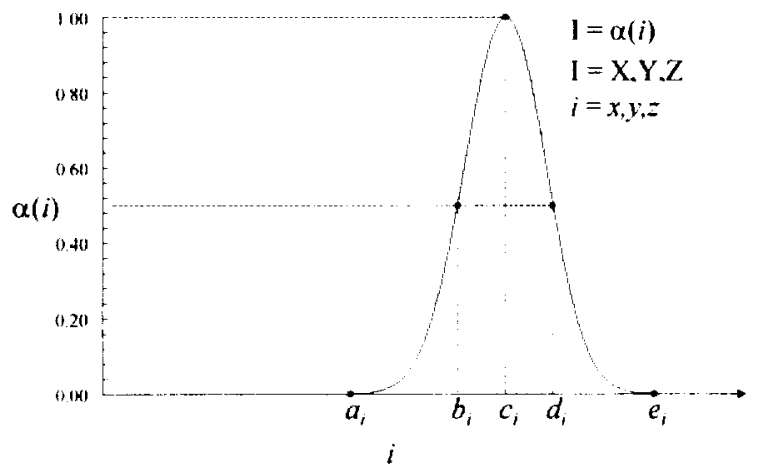

Figure 2. Discretized membership function for vertex method $(\alpha=0,0.5,1.0)$

The bounds on the membership functions for $\mathrm{X}$ and $\mathrm{Y}$ at $\alpha=0.0,0.5$ and 1.0 are $\left(a_{x}, e_{x}\right)$ and $\left(a_{y}, e_{y}\right),\left(b_{x}, d_{x}\right)$ and $\left(b_{1}, d_{v}\right)$, and $c_{x}$ and $c_{y}$, respectively. The output membership function for $Z$ is given by the maximum and minimum values of $f(X, Y)$ within the bounds of $\mathrm{X}$ and $Y$ at each $\alpha$-cut interval. Hence, for $\alpha=0$ using the vertex method, the upper bound of $Z$ is $\max \left\{f\left(a_{x}, a_{y}\right), f\left(a_{x}, e_{y}\right), f\left(e_{x}, a_{y}\right), f\left(e_{x}, e_{y}\right)\right\}$ and the lower bound is $\min \left\{f\left(a_{x}, a_{y}\right), f\left(a_{x}, e_{y}\right), f\left(e_{x}, a_{y}\right), f\left(e_{x}, e_{y}\right)\right\}$. This is repeated for the correspond-ing bounding values at each $\alpha$-cut level.

Consider the fuzzy numbers $\mathrm{X}$ and $\mathrm{Y}$ shown in Figures 3 and 4 . These two fuzzy numbers can be defined over the real field using membership functions. The membership functions for $\mathrm{X}$ and $\mathrm{Y}$ are

$$
\alpha(x)=\left\{\begin{array}{cl}
x-1, & 1 \leq x \leq 2 \\
3-x, & 2 \leq x \leq 3 \\
0, & \text { otherwise }
\end{array}\right\}
$$

and

$$
\alpha(y)=\left\{\begin{array}{cc}
\frac{(y-1)}{2}, & 1 \leq y \leq 3 \\
4-y, & 3 \leq y \leq 4 \\
0, & \text { otherwise }
\end{array}\right\}
$$

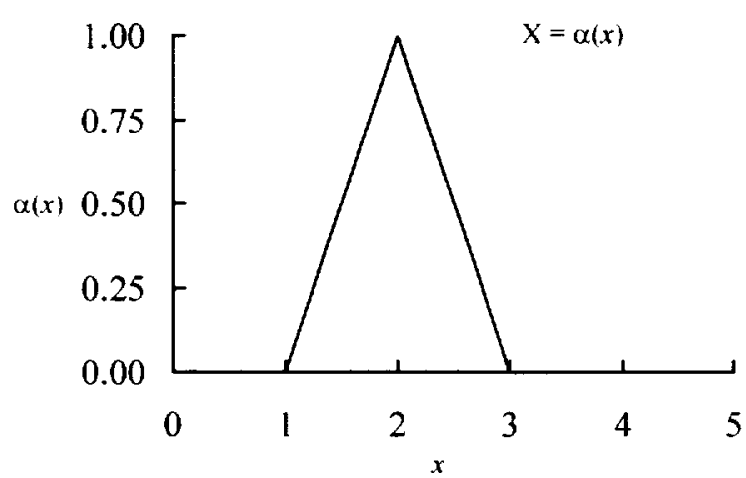

Figure 3. Membership function for $\mathrm{X}$

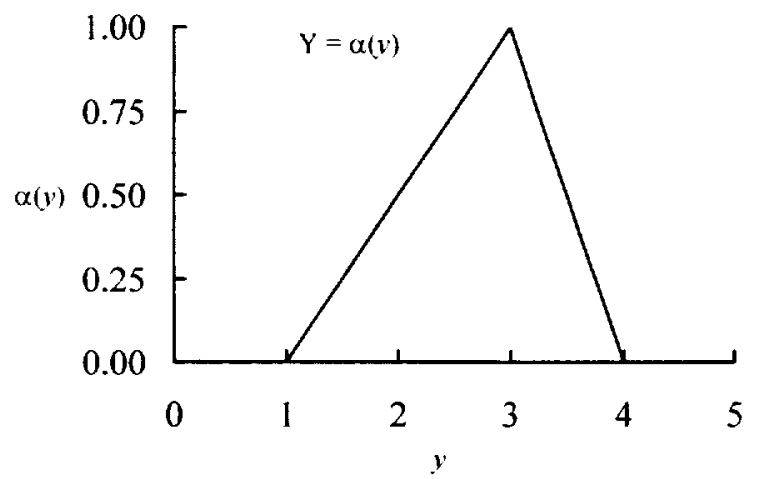

Figure 4. Membership function for $Y$

Suppose that $Z=Y-X$. The membership function $Z$ can be calculated by performing the operation for the bounds defined by a set of $\alpha$-cuts. For example let us use $\alpha$-cuts at $\alpha=0.0,0.5$ and 1.0. On $\alpha=0.0$ the bounds on the membership functions $\alpha(x)$ and $\alpha(y)$ are $(1,3)$ and $(1,4)$, respectively. To calculate $Z=Y-X$ on $\alpha=0.0$ all combinations of the bounds must be considered. Thus the set that defines the membership function of $\alpha(z)$ for $\alpha=0$ is $[\min \{(1-1),(1-3),(4-1),(4-$ 
$3)\}, \max \{((1-1),(1-3),(4-1),(4-3)\} \mid$. This yields the set $[-2,3]$ for the response $Z$ for $\alpha=0$. The procedure is repeated for $\alpha=0.5$. The bounds at $x=0.5$ are $(2,3.5)$ for $\alpha(y)$ and $(1.5,2.5)$ for $\alpha(x)$. Thus the set that defines the response membership function $Z$ for $\alpha=0.5$ is $[\min \{(2-$ $1.5),(2-2.5),(3.5-1.5),(3.5-2.5)\}, \max \{(2-1.5),(2-$

$2.5),(3.5-1.5),(3.5-2.5)\}]=[-0.5,21$. Because the membership functions $\alpha(x)$ and $\alpha(v)$ are single valued at $\alpha=1.0$ the response membership function is single valued as well. Hence, the most likely value of $Z$ occurs at $y=1.0$. The final membership function for $Z$ is shown in Figure 5.

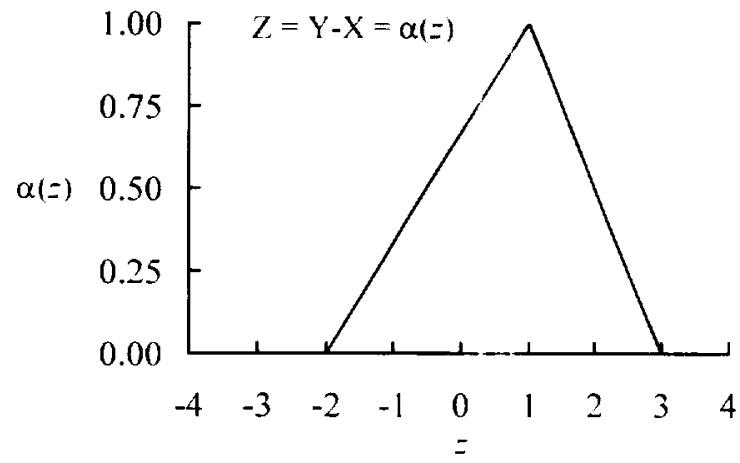

Figure 5. Membership function for $Z=Y-X$.

Dong and Shah (Ref 3$)$ noted that the maximum or minimum value of the response function might not occur at the bounds of the interval. The extremum of the function within the interval must be included or an incorrect result will be obtained. For example consider the fuzzy function $Z=X Y(4-Y)$. Where $X$ and $Y$ are shown in Figures 3 and 4 and are described by Equations $I$ and 2. respectively. The function $\mathrm{Z}$ has a maximum value that occurs when $y=2$ and $x$ takes on its maximum value. Thus to evaluate the membership function of $Z$ the value of $y$ corresponding to the extreme value of $Z$ must be considered, as well as the bounds of each $\alpha$-cut. In Figure 6 the membership function of $Z$ is shown. The solid line in Figure 6 is the membership function that is obtained when the internal extrema is considered. The dashed line in the figure is the result that would have been obtained if the internal extrema had not been considered. To obtain the correct result the global extrema must be accounted for.

One method for handling extremum interior to the intervals on each $\alpha$-cut would be to use the internal points from the $\alpha$-cut levels above the current $\alpha$-cut level. For example consider two membership functions discretized as the membership function shown in Figure 2. The calculation of possibility at $\alpha=0.0$ requires the evaluation of all the possible combinations of points from all $\alpha$-cut levels. The combinations for each $\alpha$-cut are shown in Table 1. In Table 1 the closed circular

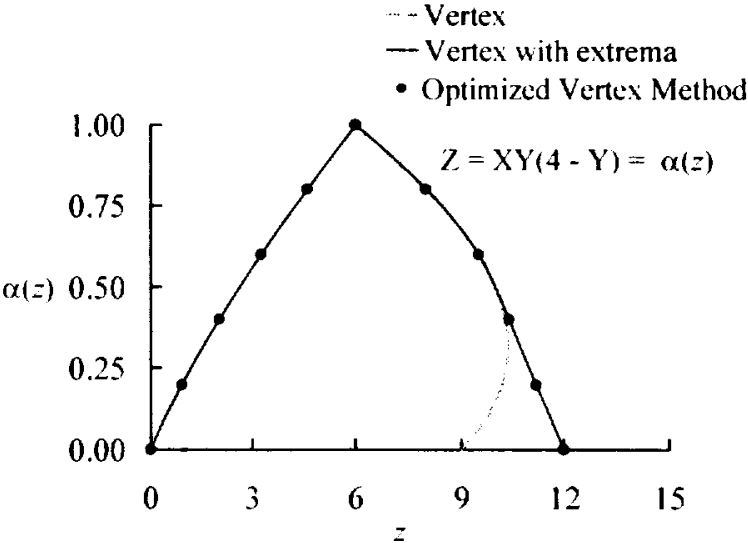

Figure 6. Membership function for $Z=X Y(4-Y)$

symbols are the points needed for the evaluation of the response at $\alpha=0.0$, the closed square symbols are the points needed at $\alpha=0.5$, and the open triangular symbol is the point needed at $\alpha=1.0$. The minimum and maximum of the system response for these points would define the response membership function at each $\alpha$-cut level. In general, for any number of $\alpha$-cuts, the function is evaluated for all the possible combinations of the interior points from all the $\alpha$-cuts. Then, the evaluation points are partitioned for each $\alpha$-cut level, and the maximum and minimum values of the response are selected.

For convex membership functions that are single valued at $\alpha=1.0$, the number of function evaluations required increases with

Table 1. Partitioning of the evaluation points to obtain the response membership function

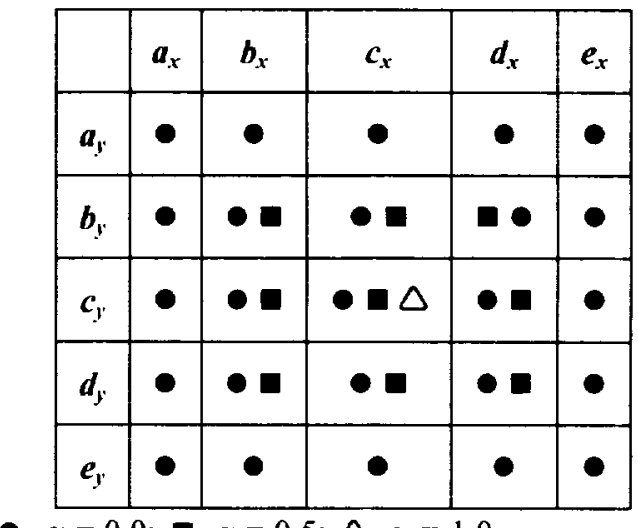

$\bullet, \alpha=0.0 ; \mathbf{\square}, \alpha=0.5 ; \Delta, \alpha=1.0$

the number of $\alpha$-cuts and the number of variables (see Figure 7). For $m$ variables and $n \alpha$-cuts this solution requires $\mathrm{m}^{2 n-1}$ function evaluations. The number of function evaluations can become unmanageable for problems with large numbers of random variables or for 
functions that change rapidly over the values of the fuzzy variables.

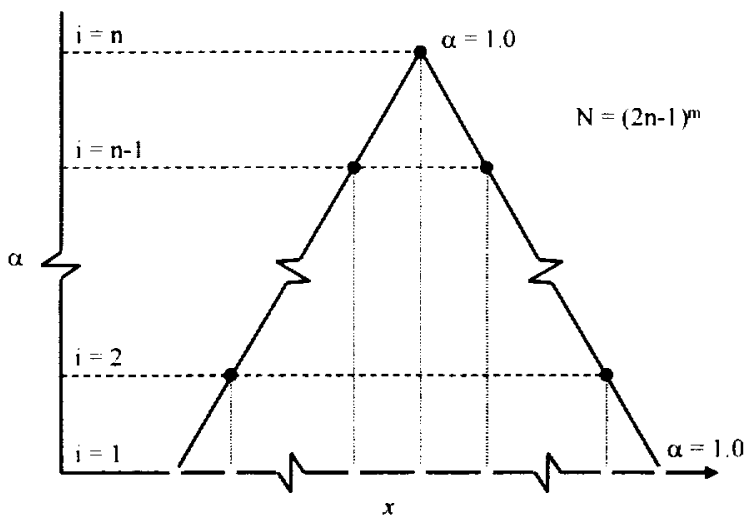

Figure 7. Alpha-cuts for vertex method including interior points

Furthermore, if an insufficient number of cuts are used the internal extremum might still be missed. Hence, a method that reduces the number of function evaluations and ensures that the solution contains the extremum of the function within the bounds of the fuzzy variables is desired. Such a method is described below.

\section{Optimized Vertex Method}

To reduce the number of calculations required to perform a possibilistic analysis the maximum and minimum values of the response function must be determined over the range of the fuzzy variables. This is accomplished by finding the extremum of the function within the interval of fuzzy variables defined by an $\alpha$-cut. In general, for complex problems, no closed form solution is available for the system response and the extremum cannot be solved for directly. However, this problem is an unconstrained optimization problem with bounded input parameters. A problem of this type can be solved using a gradientbased optimization method. In this paper the BFGS algorithm is used (Refs. 7-10).

The procedure followed in the Optimized Vertex Method (OVM) is shown in Figure 8. The upper and lower bounds on the random variables are determined from the membership functions at $\alpha=0$. The initial values of the random variables are set to their most likely values (the values that correspond to a possibility of unity). The bounds and the initial values are used as the input to the gradient-based optimization routine. Two optimizations are conducted. First, the system response is maximized. Then, the system response is minimized. The minimum and maximum system response correspond to the

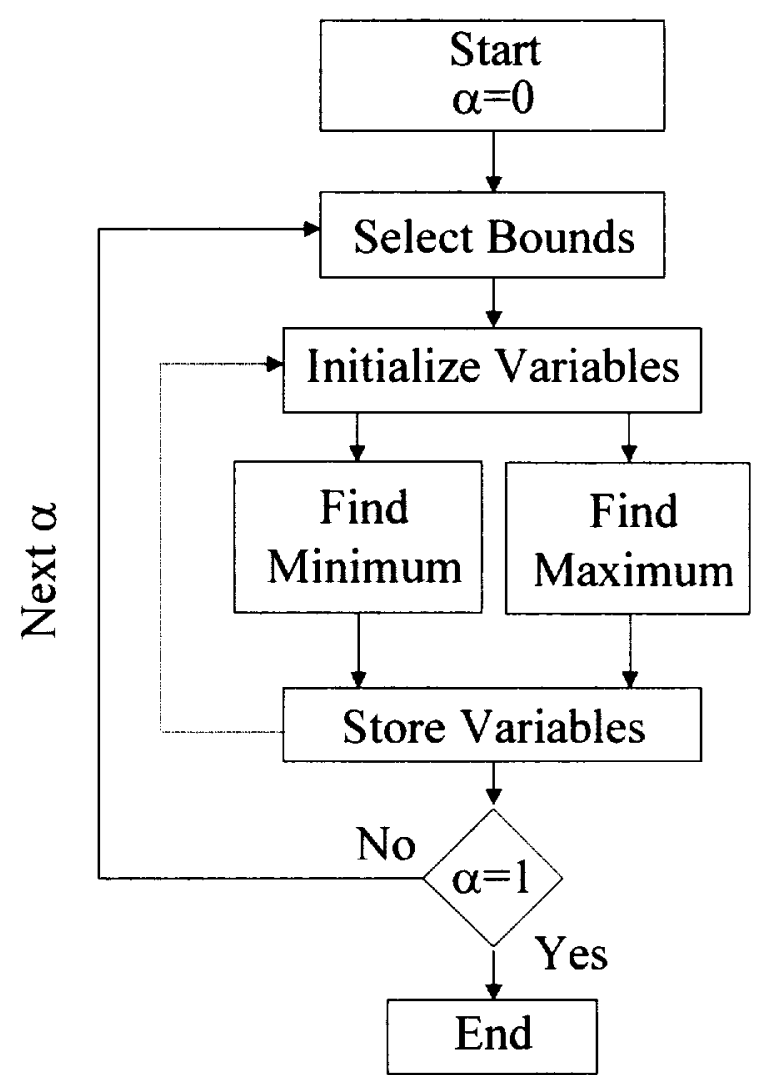

Figure 8. Procedure for optimized vertex method

upper and lower bounds of the response membership function, respectively. The values of the random variables that correspond to the minimum and maximum are stored for use in the next optimization. The bounds on the random variables are determined for the next $\alpha$-cut. On the next pass through the optimization routine the initial values for the random variables are set to the values obtained from the previous optimization. When those results are outside the bounds of the random variables, the closest bounding value is selected. The minimum and maximum responses are then obtained for next $\alpha$-cut. The procedure is repeated until all $\alpha$-cuts have been evaluated.

Consider the fuzzy numbers $\mathrm{X}$ and $\mathrm{Y}$ (Equations 1 and 2 and Figures 3 and 4, respectively) and the fuzzy function $Z=X Y(4-Y)$ once more. Applying the OVM to solve for the response membership function $\mathrm{Z}$ yields the open circular symbols shown in Figure 6. Note that OVM recovers the exact response membership function at the discrete $\alpha$-cuts. 


\section{Possibility Based Hybrid Reliability}

In References 4-6 it was noted that for single failure modes or for parallel systems the possibility of failure is conservative with respect to probability of failure. In Reference 6 the authors showed that if the fuzzy membership function for a random variable is based on the mean and standard deviation of a probabilistic random variable, the possibility of failure is one when the probability of failure is fifty-percent. Possibility effectively implies a factor of safety of two for the analysis. In the early stages of design this conservatism would certainly ensure performance, but could adversely effect the optimization of the weight of the structure.

In this paper a Bayesian approach was adopted to reduce the conservatism of possibility theory. In general when a Bayesian approach is adopted, expert knowledge is used to construct the probability density functions for the probabilistic analysis. The selection of the type of the distribution, mean value, and standard deviation for a quantity is objective, rather than subjective. In the possibility-based approach to reliability the fuzzy response of the system is used to create the probability density functions for the probabilistic analysis. First, the membership function of the system response is determined using OVM. Then, the fuzzy membership function for the system response is transformed into a probability density function. A simple integration of is performed to obtain the probability of failure.

A triangular fuzzy membership function is shown in Figure 9. The equation for this membership function is

$$
\alpha(x)=\left\{\begin{array}{cc}
\frac{x-a}{c-a}, & a \leq x \leq c \\
\frac{b-x}{b-c}, & c \leq x \leq b \\
0, & \text { otherwise. }
\end{array}\right\}
$$

Equation 3 can be transformed to a probability density function (PDF) by scaling the function by the area under the function.

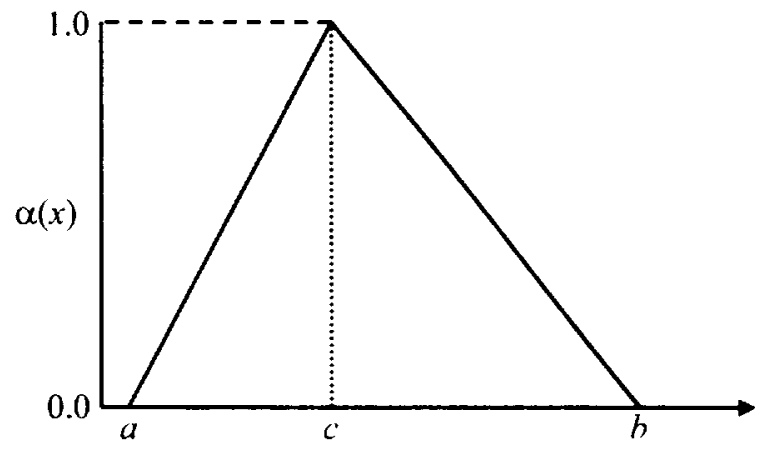

Figure 9. Triangular membership function with upper and lower bounds and most likely value

The PDF created from the transformed triangular membership function is

$$
p(x)=\left\{\begin{array}{cl}
\frac{K(x-a)}{(c-a)}, & a \leq x \leq c \\
\frac{K(b-x)}{(b-c)}, & c \leq x \leq b \\
0, & \text { otherwise }
\end{array}\right\}
$$

where

$$
K=\frac{2}{(b-a)}
$$

The cumulative distribution is obtained by integrating Equation 4. The triangular PDF has a cumulative distribution that is given by

$$
P(x)=\left\{\begin{array}{cl}
0, & x \leq a \\
\frac{(x-a)^{2}}{(b-a)(c-a)}, & a \leq x \leq c \\
\frac{(c-a)}{(b-a)}+\frac{x(2 b-x)-c(2 b-c)}{(b-a)(b-c)}, & c \leq x \leq b \\
1, & x \geq b .
\end{array}\right\}
$$

Given two fuzzy numbers, $X$ and $Y$, the probability that $X$ is greater than $Y$ is calculated by transforming $X$ and $Y$ into PDFs and evaluating the integral expression (Figure 10)

$$
p_{f}=\int_{-\infty}^{\infty} \int_{-\infty}^{x \geq y} p(\mathrm{x}) p(\mathrm{y}) d x d y
$$


Equation 7 can be evaluated directly or determined numerically. In general this procedure is not limited to triangular membership functions an can be applied to any piece-wise continuous fuzzy membership function.

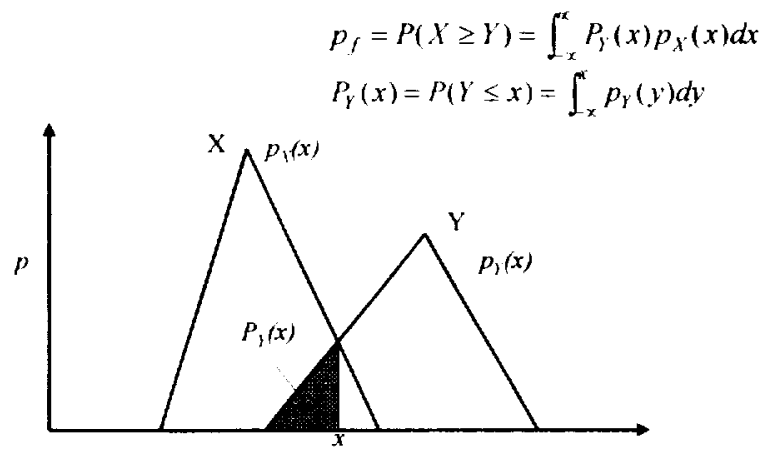

Figure 10. Probability of $X>Y$

\section{Bonded Joint}

Next, OVM is applied to calculate the possibility of failure for the problem of a bonded joint. This problem was investigated earlier by Stroud, et al. (Ref. 6). In Reference 6 two failure modes were considered, failure in the strap due to yielding and failure in the adhesive due to crack growth. In the current analysis only failure in the adhesive is considered. First, OVM is used to determine the system response. Linear and nonlinear finite-element analyses of the joint are used to obtain the response membership function for the total strain-energy release rate of a crack in the adhesive of the joint. This membership function is compared with the fracture toughness of the adhesive, which is also random. The possibility of failure, the possibility that the total strainenergy release rate is greater than the fracture toughness, is determined. Then the membership functions for total strain-energy release rate and fracture toughness are transformed into probabilitydensity functions and the probability of failure is evaluated by integrating Equation 7 explicitly. The results obtained are compared to the results obtained in Reference 6.

\section{Analysis}

A bonded joint with a crack in the bond-line is shown in Figure 11. A probabilistic and possibilistic analysis of this joint was conducted Stroud, et al (Ref. 6). Determining failure in the adhesive is accomplished by calculating the total strain-energy release rate for the crack in the adhesive of the bonded joint. Finite-element analysis is used to obtain the forces and displacements ahead of and behind the crack tip and the total strain-energy release rate is calculated using the virtual crack closure technique (Ref. 6,11). Failure is said to have occurred when the total strainenergy release rate has exceeded the fracture toughness of the

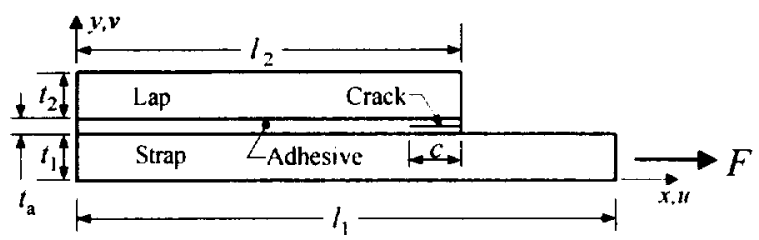

Figure 11. Bonded lap joint

adhesive. The response of the system is the total strainenergy release rate.

Table 2 contains the parameters that define the bonded joint configuration and the randomness of the variables. In Table 2 the yield stress and the critical strain-energy release rate (fracture toughness) are shown as random variables. While these limit criteria, fracture toughness and yield stress, are random they are not needed for the determination of the system response. Hence, only eight random variables are used in the evaluation of the system response.

The possibilistic analysis of the joint in Reference 6 was conducted using the vertex method for $\alpha$-cuts at $\alpha=0.0,0.5$ and 1.0 for eight random input variables. In Reference 6 response membership functions were obtained for the maximum stress in the strap of the joint and the total strain-energy release rate. The analysis required 390,625 finite-element analyses of the model to determine each response membership functions. Both nonlinear and linear analyses were conducted.

For this paper the bonded joint problem was solved using OVM and the optimization step was implemented using DOT (Ref. 12). Alpha-cuts were made at $\alpha=0.0,0.2,0.4,0.6,0.8$ and 1.0. A FORTRAN code was developed and was linked to an ABAQUS finite-element model of the bonded joint. This code was also linked to the post processing and optimization routines. The random variables were initially set to their most likely values and the dual optimization at $\alpha=0.0$ was conducted. After the first $\alpha$-cut, the initial values of the random variables were selected based on previous optimizations results. The process was repeated for the remainder of the $\alpha$-cuts. Both linear and geometrically nonlinear analyses were conducted. 
Table 2. Values of quantities that define the single lap shear joint

\begin{tabular}{|c|c|c|c|c|c|}
\hline \multirow[t]{2}{*}{ Variable } & \multirow[t]{2}{*}{ Symbol } & \multicolumn{2}{|c|}{$\begin{array}{l}\text { Probabilistic Analysis } \\
\text { (normal distributions) }\end{array}$} & \multicolumn{2}{|c|}{$\begin{array}{l}\text { Possibilistic Analysis } \\
\qquad \alpha=0\end{array}$} \\
\hline & & Mean & $\begin{array}{c}\text { Standard } \\
\text { Deviation }\end{array}$ & $\begin{array}{l}\text { Lower } \\
\text { Bound } \\
\end{array}$ & $\begin{array}{c}\text { Upper } \\
\text { Bound } \\
\end{array}$ \\
\hline Thickness, strap - in. & $t_{1}$ & 0.125 & 0.005 & 0.11 & 0.14 \\
\hline Thickness, lap - in. & $t_{2}$ & 0.125 & 0.005 & 0.11 & 0.14 \\
\hline Length, strap - in. & $l_{1}$ & 12.0 & 0 & 12 & 12 \\
\hline Length, lap - in. & $l_{2}$ & 10.0 & 0.16 & 9.52 & 10.48 \\
\hline Thickness, adhesive - in. & $t_{\mathrm{a}}$ & 0.0050 & 0.0005 & 0.0035 & 0.0065 \\
\hline Length, crack - in. & $c$ & 4.00 & 0.08 & 3.76 & 4.24 \\
\hline Modulus, metallic adherends - psi & $E_{\mathrm{m}}$ & $10,500,000$ & 105,000 & $10,185,000$ & $10,815,000$ \\
\hline Poisson's ratio, metallic adherends & $v_{\mathrm{m}}$ & 0.3125 & 0 & 0.3125 & 0.3125 \\
\hline Modulus, adhesive - psi & $E_{\mathrm{a}}$ & 336,000 & 16,800 & 285.600 & 386,400 \\
\hline Poisson's ratio, adhesive & $v_{\mathrm{a}}$ & 0.40 & 0 & 0.4 & 0.4 \\
\hline Fracture Toughness - in. $\mathrm{lb}^{\prime}$ in. $^{2}$ & $G_{c}$ & 5.50 & 0.66 & 3.52 & 7.48 \\
\hline Yield stress 2024 T3 - psi & $\sigma_{\text {yield }}$ & 44,000 & 880 & 41.360 & 46,640 \\
\hline Crack Growth Increment - in. & $\Delta c$ & 0.00125 & 0 & 0.00125 & 0.00125 \\
\hline
\end{tabular}

In Figures 12 and 13 the response membership functions for the total strain-energy release rate are compared to the same result obtained in Reference 6. Figure 12 shows the results for the linear analysis and Figure 13 shows the result for the nonlinear analysis. To obtain the response membership functions 554 and 514 finite-element simulations were needed for the linear and nonlinear analyses, respectively.

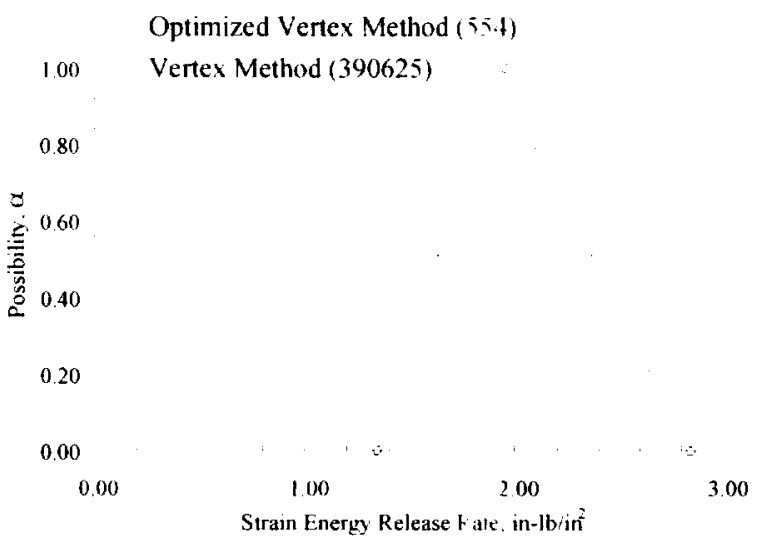

Figure 12. Response membership function from linear analysis

The sensitivity of the total strain-energy release rate to changes in the random variables is shown in Figures 14 and 15. The derivative values are obtained directly from the optimization routine used in

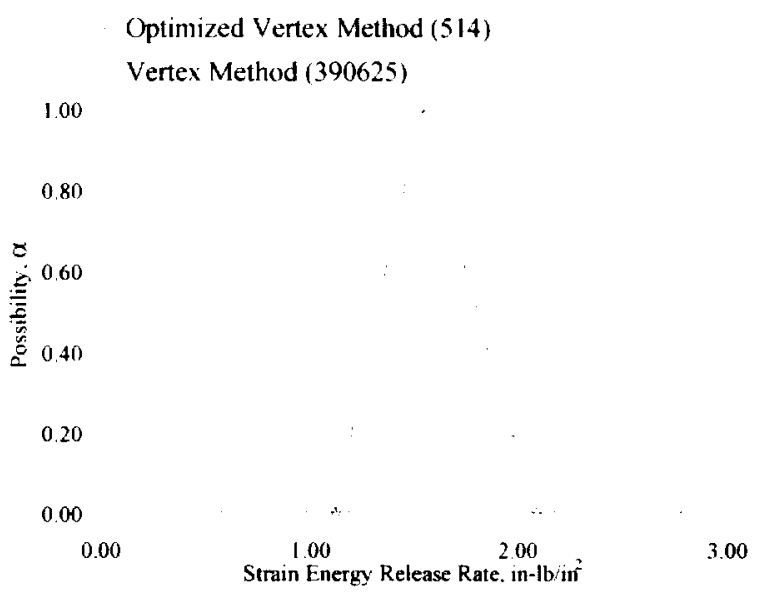

Figure 13. Response membership function from nonlinear analysis

the calculation of the output membership function. They are obtained from a central difference calculation about the most likely value of the system response. They could also be shown for each point on the bounds of the response membership function. In this instance the sensitivity is defined as the derivative of the response with respect to the random variable multiplied by the standard deviation of that random variable. Note that these sensitivities can be used to determine the relative importance of modeling the randomness of the input parameters. From figures 14 and 15 it is clear that 
some random variables have little effect on the system response. Design sensitivities are an added benefit of using OVM.

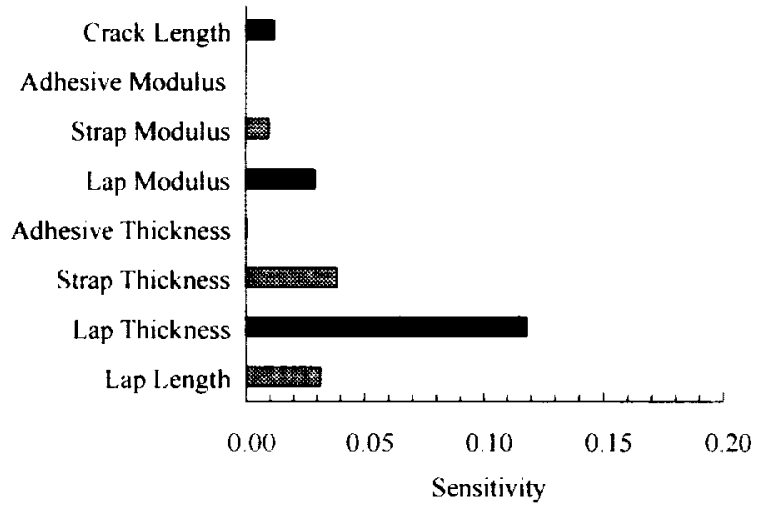

Figure 14. Design sensitivities obtained from the optimized vertex calculations for the linear analysis

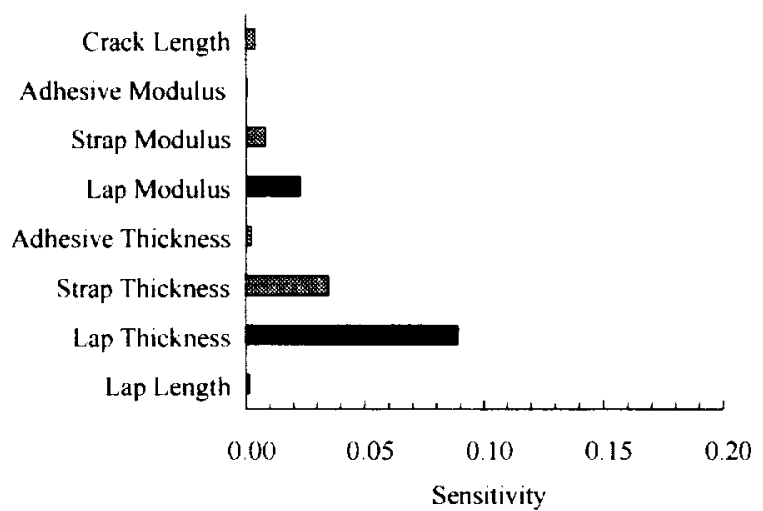

Figure 15. Design sensitivities obtained from the optimized vertex calculations for the nonlinear analysis

The possibility of failure is determined by determining the possibility that the total strain-energy release rate is greater than the fracture toughness of the adhesive, $G_{T}>G_{c}$. In this case both the $G_{T}$ and $G_{c}$ are fuzzy numbers. To demonstrate the calculation of the possibility of failure, consider the two fuzzy numbers shown in Figure 16. The possibility that $X>Y$ is the point where the two membership functions cross. This point is shown as $\alpha$ in Figure 16. Once the most likely value of $X$ is greater than the most likely value of $Y$, the possibility that $\mathrm{X}$ is greater than $\mathrm{Y}$ is unity. The probability of failure for the bonded joint was also calculated using the hybrid reliability methods discussed previously. The possibility and probability of failure for linear and nonlinear analysis of the bonded joint are shown for various loads in Figures 17 and 18, respectively. In these figures the probability of failure obtained in Reference 6 is included for comparison.

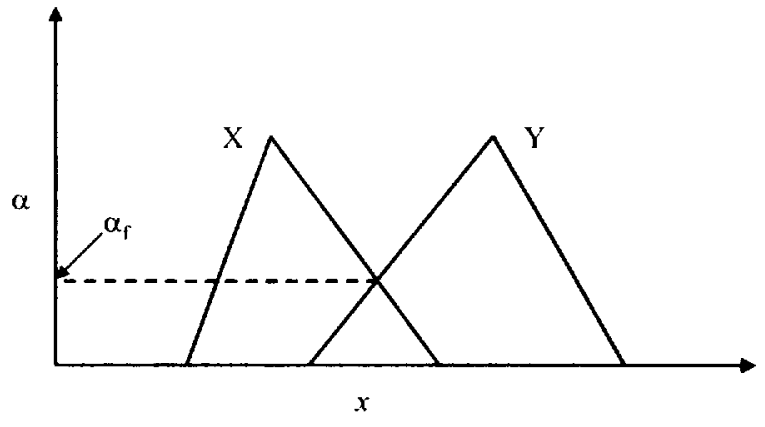

Figure 16. Possibility of $X>Y$

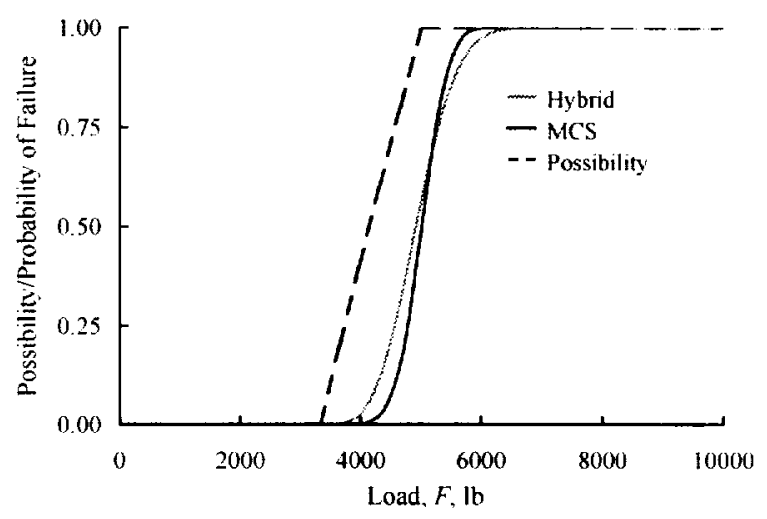

Figure 17. Possibility and probability of failure obtained from linear analysis

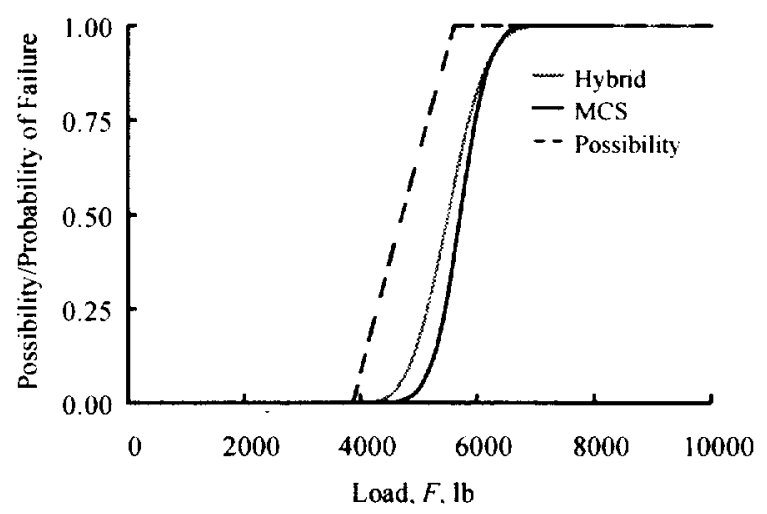

Figure 18. Possibility and probability of failure obtained from nonlinear analysis.

\section{$\underline{\text { Discussion }}$}

The optimized vertex method (OVM) significantly reduces the number of calculations required to obtain the system response. However, the maximum and minimum response of the bonded joint on each $\alpha$-cut corresponded to the bounds of the random variables. This means that for the bonded joint 
problem there were no internal extrema that needed to be considered. If this could have been determined initially, only bounding values of the random variables would have been needed to obtain the system response membership function. The number of function evaluations would have been reduced to $256\left(2^{\mathrm{m}}\right)$ for each $\alpha$-cut. Conversely, there could have been multiple extrema within the bounds of the random variables. In this case the gradient-based optimizer could have been trapped at a local minimum or maximum of the response and obtained an incorrect solution. Since the response was calculated using both the vertex and optimized vertex, the solution obtained was the correct one, but the underlying problem with optimization was not addressed. To avoid being trapped in local extrema a Tunneling method (Ref. 13) could be adopted for the optimization step to obtain the global minimum or maximum of the response. However, the Tunneling method is not available in DOT.

As was shown in Reference 6, the possibility of failure for the bonded joint was conservative in comparison to the probability of failure (Figure 17 and 18). The possibility based hybrid approach obtained close correlation to the reliability solution from MCS. This result is somewhat artificial as the membership functions were constructed based on the mean and standard deviation used for probability density functions given in Reference 6. However, the fact that a good approximation was obtained may be useful for cases where a probabilistic analysis is not possible due to either lack of information or when time is critical. The OVM approach may be appropriate for the early stages of design while a full reliability assessment using MCS should be conducted at the end of the design process.

\section{Concluding Remarks}

A method of calculating the fuzzy response of a system is presented. This method, called the Optimized Vertex Method (OVM), is based upon the vertex method but requires much fewer function evaluations. Improved efficiency is obtained through the use of optimization to find the minimum and maximum of the system response on a defined interval of the random variables.

The method is validated for a simple mathematical function and demonstrated by calculating the response membership function of strain-energy release rate for a bonded joint with a crack. Eight random variables were considered. The response membership functions obtained from OVM reproduced the values obtained using the vertex method in less than 1,000 simulations compared to more than 390,000 required for the vertex method. The possibility of failure of the bonded joint was determined over a range of loads.

After completing the possibilistic analysis, the possibilistic (fuzzy) membership functions were transformed to probability density functions. Using the transformed membership functions the probability of failure of the bonded joint was calculated. This approach is called a possibility based hybrid reliability assessment. The possibility and probability of failure are presented and compared to a Monte Carlo Simulation (MCS) of the bonded joint. The possibility based-hybrid reliability method yielded results very close to those obtained from the MCS.

\section{$\underline{\text { References }}$}

1. Cappelle, B. and Kerre, E. E., (1995), "Computer assisted reliability analysis: An application of possibilistic reliability theory to a subsystem of a nuclear power plant," Fuzzy Sets and Systems, Vol. 74, pp. 103-113.

2. Cremona, C. and Gao. Y.. (1997), "Possibilistic reliability theory: theoretical aspects and applications," Structural Safety, Vol. 19, No. 2, pp. 173-201.

3. Dong, Weimin and Shah, Haresh C., (1987), "Vertex method for computing functions of fuzzy variables," Fuzzy Sets and Systems, Vol. 24, pp. 65-78.

4. Ferrari, Paola and Savoia, Marco, (1998), "Fuzzy number theory to obtain conservative results with respect to probability," Comput. Methods Appl. Mech. Engrg., Vol. 160, pp. 205-222.

5. Nikolaidis, Efstratios; Cudney, Harley; Chen. Sophie; Haftka, Raphael T.; and Rosca, Raluca, (1999), "Comparison of Probabilistic and Possibility Theory-Based Methods for Design Against Catastrophic Failure Under Uncertainty," Proceedings of the 1999 ASME Design Engineering Technical Conferences,

$11^{\text {th }}$ International Conference on Design Theory and Methodology, Sept. 12-15, 1999 , Las Vegas, Nevada. Available as DTC99/DTM-8758.

6. Stroud, W. Jefferson; Krishnamurthy, T.; and Smith, Steven A., (2001), "Probabilistic and Possibilistic Analyses of the Strength of a Bonded Joint," $42^{\text {nd }}$ AIAA/ASME/ASCE/AHS/ASC, Structures, Structural Dynamics and Materials Conference, 16-19 April, 2001, Seattle, WA, AIAA-2001-1238. 
7. Broyden, C.G., (1970), "The convergence of a class of double rank minimization algorithims, Parts I and II," J. Inst. Math. Appl., Vol. 6, pp. 76-90, 222-231.

8. Goldfarb, D., (1970), "A family of variable metric methods derived by variational means," Math. Comput., Vol. 24, pp. 23-26.

9. Fletcher, R., (1970), "A New Approach to Variable Metric Algorithims," Computer J., vol. 13, pp. 317-322.

10. Shannon, D.F., (1970), "Conditioning of quasi-Newton methods for function minimization," Math. Comput., Vol. 24, pp. 647-656.
11. Rybicki, E.F., and Kanninen, M.F., (1977), "A Finite-Element Calculation of Stress-Intensity Factors By A Modified Crack Closure Integral," Engineering Fracture Mechanics, Vol. 9, pp. 931-938.

12. Anonymous, (1999), "DOT, Design Optimization Tools, Users Manual, Version 5.0," Vanderplaats Research and Development, Inc., Colorado Springs, CO

13. Levy, A.V. and Montalvo, A., (1985), "The tunneling method for Global Optimization", SIAM J. of Sci. and Stat. Comp., Vol. 1, No. 15 